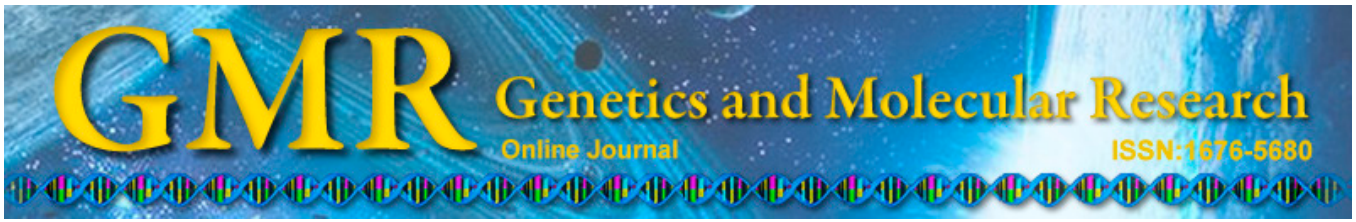

\title{
Association between growth differentiation factor-15 and chronic heart failure in coronary atherosclerosis patients
}

\author{
Z.D. Zhu and T. Sun \\ Division of Cardiology, Huashan Hospital of Fudan University, Shanghai, \\ China \\ Corresponding author: T. Sun \\ E-mail: suntaofudan@outlook.com
}

Genet. Mol. Res. 14 (1): 2225-2233 (2015)

Received January 21, 2014

Accepted August 22, 2014

Published March 27, 2015

DOI http://dx.doi.org/10.4238/2015.March.27.8

ABSTRACT. We explored the association between plasma growth
differentiation factor 15 (GDF-15) levels and chronic heart failure
(CHF) in coronary heart disease patients. We measured plasma GDF-15
and N-terminal pro-B-type natriuretic peptide (NT-proBNP) levels in
269 untreated coronary heart disease patients ( 98 with CHF, 84 without
CHF, and 87 control patients) using an enzyme-linked immunosorbent
assay. All subjects were examined by echocardiography and left
ventricular ejection fraction. We found that plasma GDF-15 levels in
coronary atherosclerosis patients with CHF [median 1622.48 (25-75th
percentile: $887.53-1994.93)$ ng/L] were higher than those in coronary
atherosclerosis patients without CHF [ 944.99 ( $856.12-999.78)$ ng/L]
and control patients (P $<0.05$ ). NT-proBNP showed the same trend
as GDF-15. We also used the New York Heart Association functional
classification to subgroup CHF patients and found that the GDF-15
level was higher in all subgroup patients with CHF. After adjusting
for covariates, plasma GDF-15 levels were found to be positively
related to NT-proBNP ( $\mathrm{r}=0.861, \mathrm{P}<0.001)$ and negatively related to 
left ventricular ejection fraction $(\mathrm{r}=-0.936, \mathrm{P}<0.001)$. Furthermore, receiver operating characteristic curves of GDF-15 and NT-proBNP were constructed and the area under the curve for the untransformed GDF-15 and NT-proBNP was 0.804 and 0.795 , respectively. Plasma GDF-15 levels and NT-proBNP are associated with CHF in coronary atherosclerosis patients and can be used as biomarkers.

Key words: Association analysis; Chronic heart failure; ROC curve; Growth differentiation factor-15; N-terminal pro-brain natriuretic peptide

\section{INTRODUCTION}

With changes in lifestyle and dietary habits, cardiovascular disease has become increasingly prevalent. Among the cardiovascular diseases, coronary artery atherosclerosis is the leading cause of deaths worldwide. Chronic heart failure (CHF) is caused by coronary artery atherosclerosis and is the most common form of heart failure. CHF has become an epidemic disease in recent years. Developing new methods for treating CHF is an urgent need. Biomarkers that can reflect the diagnosis, risk classification, prognosis, symptoms, and outcome of the disease are valuable for disease identification. N-terminal pro-B-type natriuretic peptide (NT-proBNP) belongs to the natriuretic peptide family and is an established biomarker for the diagnosis and prognosis of CHF. Bootcov et al. (1997) first reported growth differentiation factor 15 (GDF-15) as a new member of transforming growth factor- $\beta$ family in 1997; this molecule can regulate the growth and suppress metastasis of tumor cells, and thus is known as macrophage inhibitory cytokine-1 (Tan et al., 2000). Recently, GDF-15 was found to have a protective effect oncoronary artery (Kempf and Wollert, 2009) and has a close relationship with cardiovascular disease (Kempf et al., 2006; Xu et al., 2006). Furthermore, several multicenter clinical trials showed that GDF-15 can be used as a biomarker of cardiovascular disease (Wollert et al., 2007a,b; Khan et al., 2009; Kempf et al., 2009, 2011) and is of independent prognostic value in predicting acute coronary symptom and CHF (Kempf et al., 2007a,b). However, the relationship between GDF-15 and the pathophysiology of cardiovascular disease has not been thoroughly analyzed. In this study, we examined the association between GDF-15 and CHF in coronary atherosclerosis patients and explored its value for CHF diagnosis.

\section{MATERIAL AND METHODS}

\section{Study population}

We studied 269 consecutive coronary angiography patients admitted to the Cardiology Department of Huashan Hospital, Fudan University, Shanghai, China. Patients were recruited between February 1, 2010 and March 31, 2013. Among the patients, 98 were CHF patients (68 males, 30 females, with an average age of $65.87 \pm 14.21$ years); these patients comprised the CHF group. CHF diagnosis was based on the revised Framingham criteria in 1997 (Hunt, 2005). The cardiac functions of patients were classified according to the New York Heart Association (NYHA) criteria (33 in Class I, 31 in Class II, 16 in Class III, and 18 in Class IV for all 98 CHF patients). Significant coronary artery stenosis was defined as $>50 \%$ narrowing of 
single or multi-vessels. Eighty-four patients were confirmed to have coronary artery stenosis and were enrolled in the coronary artery disease (CAD) group. Eighty-seven patients with stenosis from $0-50 \%$ were enrolled as the control group. All patients provided written informed consent to participate in the study. All participants were of Han Chinese nationality.

Exclusion criteria included: refusal to provide consent, acute heart failure 2 weeks before blood sampling, primary and secondary myocardiopathy, combination with congenital heart disease, stroke within the past 12 months, combination with peripheral arterial disease, inflammatory disease or autoimmune disease, active infection or tumor, serum creatinine $>180$ $\mu \mathrm{M}$, combination with chronic bronchitis, pulmonary emphysema, and asthma.

Blood samples of the enrolled patients were collected and analyzed for routine blood testing, blood glucose, total plasma cholesterol, triglyceride, high-density lipoprotein cholesterol, low-density lipoprotein cholesterol, creatinine, uric acid, blood urea nitrogen, and highsensitivity C-reactive protein (CRP).

\section{Blood sampling and cytokine detection}

From the patients, $3 \mathrm{~mL}$ blood was collected from the median cubital vein of the patient into a tube containing anticoagulant. After incubation at room temperature for $2 \mathrm{~h}$, the blood was centrifuged at $3000 \mathrm{~g}$ for $10 \mathrm{~min}$. The serum separated from the blood was stored at $-80^{\circ} \mathrm{C}$ until processing. Enzyme-linked immunosorbent assay kits from USCN Life Science, Inc. (Hubei, China) were used to detect GDF-15 and NT-proBNP.

\section{Echocardiography measurement}

Echocardiography was performed for all enrolled patients (iE33; Phillips Healthcare, Amsterdam, Netherlands). Patients were examined by a professional sonographer in a quiet room at constant temperature. No clinical profiles or biochemical data were revealed to the sonographer. The data were measured according to the criteria of the American Heart Association, American College of Cardiology, and American Society of Echocardiology (Cheitlin et al., 2003). M-dimensional echocardiography was recorded for the left ventricular ejection fraction (LVEF).

\section{Coronary angiography measurement}

The right and left coronary artery was processed for angiography measurement according to the Judkins criteria. Angiograms were evaluated by 2 experienced cardiologists and quantification software. Significant coronary artery stenosis was defined as $\geq 50 \%$ narrowing of the vessel lumen.

\section{Statistical analyses}

All statistical analyses were performed using the SPSS 13.0 (SPSS, Inc., Chicago, IL, USA) or the SigmaStat (Systat, Chicago, IL, USA) software. The data are presented as the percentage, median (25-75th percentile) or means \pm standard deviation as indicated. The measurement data and counting data are reported using means \pm SD and percentages, respectively. The Student $t$-test and $\chi^{2}$ test were used to analyze measurement data and count data, re- 
spectively. Pearson's method was applied for correlation analysis. Logistic regression analysis was used to estimate risk factors. A variable selection method was based on Backward (Wald) method. The criterion of entry was $\mathrm{P}<0.05$. The results are presentedas the odds ratio (OR) and $95 \%$ confidence interval (CI). Significance was defined as $\mathrm{P}<0.05$.

\section{RESULTS}

\section{Patient demographics}

Among the 269 patients that received coronary angiography, 98 had CHF, 84 were diagnosed with coronary artery stenosis without CHF (defined as the CAD group), and 87 had stenosis from $0-50 \%$ (defined as the control group). The clinical details of patients are shown in Table 1. There were no significant differences in age, blood pressure, and blood lipids between groups $(\mathrm{P}>0.05)$. The level of high-sensitivity CRP in the CHF and CAD patients was significantly higher than that in control group.

Table 1. Clinical characteristics.

\begin{tabular}{lccc}
\hline & CHF group $(\mathrm{N}=98)$ & CAD group $(\mathrm{N}=84)$ & Control group $(\mathrm{N}=87)$ \\
\hline Age (years) & $57.6 \pm 5.7$ & $58.3 \pm 5.4$ & $56.9 \pm 5.6$ \\
Male (\%) & 77.3 & 59.5 & 70.1 \\
BMI $\left(\mathrm{kg} / \mathrm{m}^{2}\right)$ & $25.7 \pm 3.1$ & $25.9 \pm 3.4$ & $25.4 \pm 3.2$ \\
SBP $(\mathrm{mmHg})$ & $124.4 \pm 21.3$ & $126.3 \pm 24.6$ & $125.1 \pm 25.6$ \\
DBP $(\mathrm{mmHg})$ & $73.9 \pm 10.5$ & $74.0 \pm 11.7$ & $73.6 \pm 12.4$ \\
Smoker $(\%)$ & 78.0 & 79.0 & 76.0 \\
Hs-CRP $(\mathrm{mg} / \mathrm{L})$ & $10.8 \pm 28.7^{*}$ & $9.4 \pm 26.9 *$ & $2.5 \pm 5.1$ \\
TC $(\mathrm{mM})$ & $4.7 \pm 0.8$ & $4.7 \pm 0.8$ & $4.6 \pm 0.9$ \\
TG $(\mathrm{mM})$ & $2.0 \pm 1.2$ & $2.1 \pm 1.2$ & $2.0 \pm 1.3$ \\
HDL-c $(\mathrm{mM})$ & $1.0 \pm 0.2$ & $1.1 \pm 0.1$ & $1.1 \pm 0.2$ \\
LDL-c (mM) & $3.1 \pm 0.6$ & $3.0 \pm 0.7$ & $2.9 \pm 0.8$ \\
\hline
\end{tabular}

*Significant difference compared to the control group $(\mathrm{P}<0.05)$. BMI: body mass index; SBP: systolic blood pressure; DBP: diastolic blood pressure; Hs-CRP: High-sensitivity C reaction protein; TC: total cholesterol; TG: triglyceride; HDL-c: high density lipoprotein cholesterol; LDL-c: low density lipoprotein cholesterol.

\section{GDF-15 and NT-proBNP levels in the CHF, CAD, and control groups}

The plasma GDF-15 level in the CHF group [median 1622.48, 25-75th percentile (887.53-1994.93) ng/L] was significantly higher than that in the control group [median 798.45, 25-75th percentile $(728.21-872.67) \mathrm{ng} / \mathrm{L}](\mathrm{P}<0.05)$. The plasma GDF-15 level in the CAD group [median $944.99,25-75$ th percentile $(856.12-999.78) \mathrm{ng} / \mathrm{L}$ ] was also significantly higher than that in the control group $(\mathrm{P}<0.05)$. The NT-proBNP level in the CHF group [median $564.00,25-75$ th percentile $(158.00-857.00) \mathrm{ng} / \mathrm{L}]$ was significantly higher than that in the control group [median $121.00,25-75$ th percentile $(108.25-133.75) \mathrm{ng} / \mathrm{L}](\mathrm{P}<0.05)$. No difference was observed between the CAD and control groups regarding the NT-proBNP level. All data are shown in Table 2. 
Table 2. GDF-15, NT-proBNP, and LVEF in different patient groups.

\begin{tabular}{llccc}
\hline & & GDF-15 $(\mathrm{pg} / \mathrm{mL})$ & NT-proBNP(pg/mL) & LVEF $(\%)$ \\
\hline CHF group & & & & \\
$(\mathrm{N}=98)$ & $\mathrm{I}(\mathrm{N}=33)$ & $982.89(807.67-1501.22)^{*}$ & $233.00(104.75-552.00)^{*}$ & $60.00(54.75-65.00)$ \\
NYHA class & II $(\mathrm{N}=31)$ & $1614.49(810.17-1825.32)^{*}$ & $278.00(133.25-716.50)^{*}$ & $49.00(47.25-52.00)^{*}$ \\
& III $(\mathrm{N}=16)$ & $1940.46(1836.70-2062.54)^{*}$ & $790.00(636.50-1490.50)^{*}$ & $41.50(39.50-43.50)^{*}$ \\
& IV $(\mathrm{N}=18)$ & $3904.87(3690.32-4019.31)^{*}$ & $4665.00(3712.00-5442.00)^{*}$ & $35.00(31.00-37.00)^{*}$ \\
& Median $(25-75 \%)$ & $1622.48(887.53-1994.93)^{*}$ & $564.00(158.00-857.00)^{*}$ & $49.00(41.00-57.00)^{*}$ \\
CAD group $(\mathrm{N}=84)$ & & $944.99(856.12-999.78)^{*}$ & $137.50(103.00-154.00)$ & $63.00(60.00-65.00)$ \\
Control group $(\mathrm{N}=87)$ & & $798.45(728.21-872.67)$ & $121.00(108.25-133.75)$ & $64.00(62.00-67.00)$ \\
\hline
\end{tabular}

*Significant difference compared to the control group $(\mathrm{P}<0.05)$. GDF-15 = growth differentiation factor 15; NT-proBNP $=$ n-terminal pro-brain natriuretic peptide; $\mathrm{LVEF}=$ left ventricle eject fraction; $\mathrm{CHF}=$ chronic heart failure; NYHA class = New York Heart Association class; CAD = coronary artery disease.

\section{Comparison of subgroups in CHF with the CAD and control group}

GDF-15 levels in subgroups (according to NYHA classification) of CHF and the CAD group were significantly higher than that in the control group (Table 2 and Figure $1 \mathrm{~A}, \mathrm{P}<$ 0.05). NT-proBNP levels in the CHF subgroup were significantly higher than that in the control group (Table 2 and Figure 1B, $\mathrm{P}<0.05$ ).
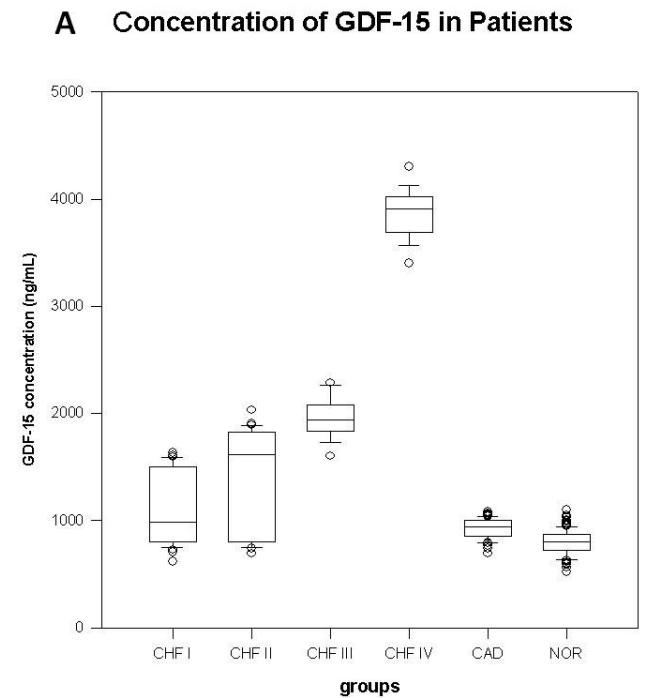

B Concentration of NT-proBNP

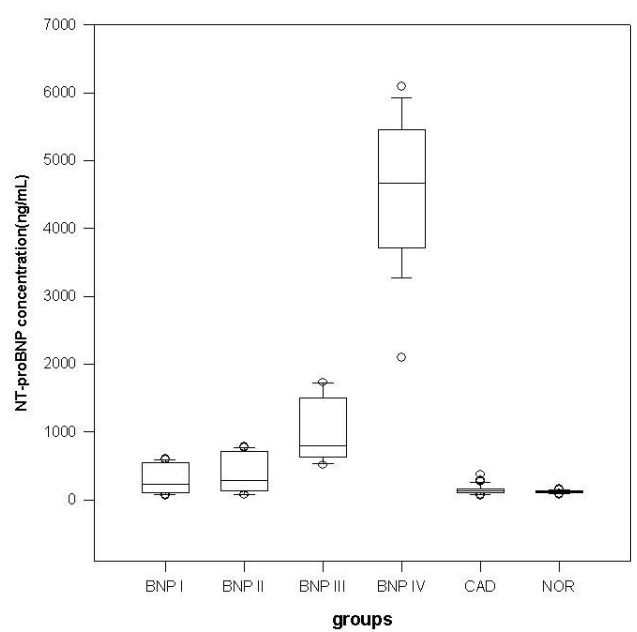

Figure 1. A. Plasma GDF-15 level in different patient groups, CHF I-IV were classified based on NYHA standards. B. Plasma NT-proBNP level in different patient groups, CHF I-IV were classified based on NYHA standards.

\section{Association between GDF-15 and risk factors in cardiovascular disease}

GDF-15 showed no association with age, gender, and body mass index. Smoking, alcohol drinking, diabetes, and hypertension were also not associated with GDF-15. GDF-15 was correlated with the NT-proBNP level $(\mathrm{r}=0.861, \mathrm{P}<0.001)$ and was negatively correlated with LVEF $(r=-0.936, \mathrm{P}<0.001)$. 


\section{Receiver operating characteristic (ROC) curve of the CHF patients}

An ROC curve of GDF-15 was constructed to discriminate between coronary atherosclerosis patients with or without CHF. The area under the curve for continuous untransformed GDF-15 was 0.804 (95\%CI, 0.739-0.869) (P<0.001). An ROC curve for NT-proBNP was also constructed and the area under the curve was $0.795(95 \% \mathrm{CI}, 0.727-0.863)(\mathrm{P}<0.001)$ (Figure 2). At the cutoff value for GDF-15 $(1086.38 \mathrm{pg} / \mathrm{mL})$, the diagnosis sensitivity and specificity were 72.4 and $93.6 \%$, respectively.

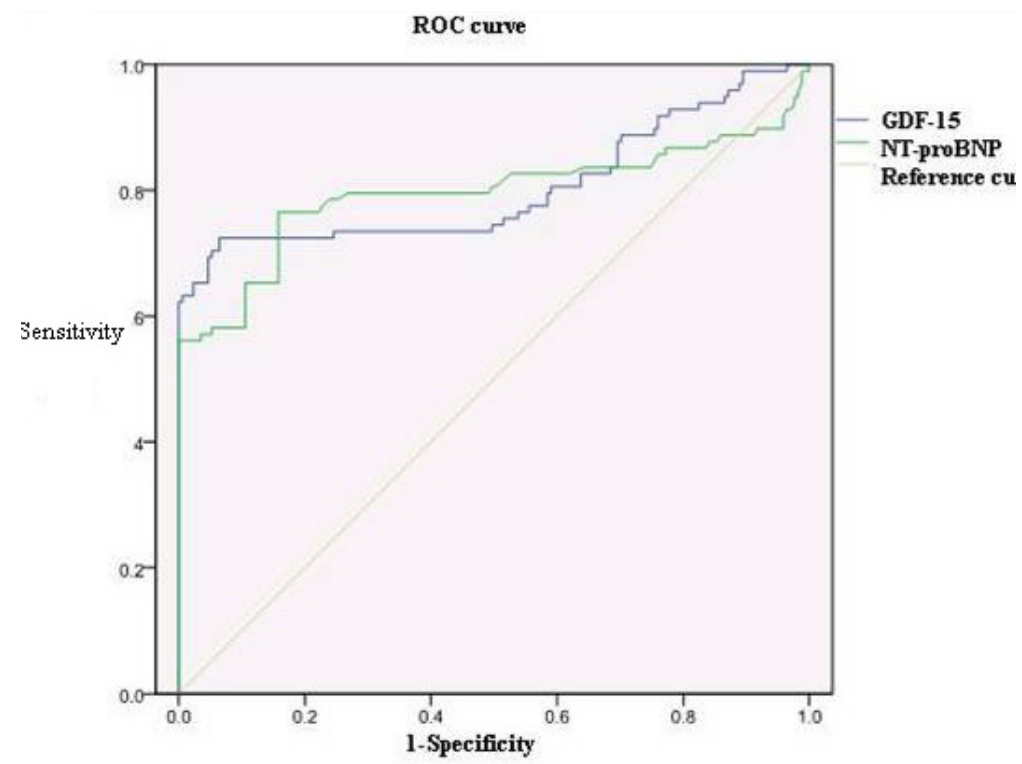

Figure 2. ROC curve for GDF-15 and NT-proBNP. GDF-15 (blue), NT-proBNP (green), and reference curve (purple).

\section{DISCUSSION}

NT-proBNP, known as a common myocardium marker, is used in the diagnosis of cardiovascular diseases. In our study, the NT-proBNP level in the CHF group was significantly higher than that in the control group. This result confirmed the value of NT-proBNP in CHF diagnosis. However, there was no difference between the CAD group and the control group, indicating that NT-proBNP is limited for CAD diagnosis. This result is consistent with those of Khan et al. (2009). In addition to NT-proBNP, other recently discovered biomarkers can be used (Iqbal et al., 2013; Lok et al., 2013), including galectin-3, ST2, mid-regional proadrenomedullin, interleukin-6, CRP, and copeptin. Among these, galectin-3 is a good prognosis biomarker for mortality (van Kimmenade et al., 2006; Lok et al., 2010; Shah et al., 2010), but it occasionally must be combined with other markers such as NT-proBNF to better predict mortality and re-hospitalization in patients (de Boer et al., 2011). Mid-regional pro-adrenomedullin and its preceding form, prepro adrenomedullin, comprise approximately $85 \%$ of systemic adrenomedullin; because the latter has a half-life of only 22 min, mid-regional proadrenomedullin has been shown to be a more practical marker for evaluating acute changes 
(Meeran et al., 1997; von Haehling et al., 2010). Because ST2 and interleukin-6 are released following multiple stimuli, multimarker combination testing is necessary (Tsutamoto et al., 1998; Sanada et al., 2007). CRP is a good biomarker for acute phase patients. It is released by the liver and plays an important role in the protein cascade caused by inflammatory stimuli (Meier-Ewert et al., 2004). Copeptin, also known as arginine vasopressin, can exacerbate fluid overload, thereby aggravating vasoconstriction, systolic and diastolic wall stress, myocyte hypertrophy, and hyponatremia in heart failure patients. However, arginine vasopressin is unstable and highly clearable, and therefore its use as a biomarker remains limited (Goldsmith and Gheorghiade, 2005; Morgenthaler et al., 2006; Szinnai et al., 2007). Identifying novel and effective biomarkers is necessary.

GDF-15 is a stress response protein of the transforming growth factor- $\beta$ superfamily. It is thought to participate in emergence and development of tumors, regulate tumor growth, and suppress tumor metastasis (Tan et al., 2000). GDF-15 has been shown to display a broad spectrum of biological activities, including protection from cardiovascular diseases and other functions. GDF-15 is one of the newest biomarkers used to determine CHF disease. Increased GDF-15 is associated with increased left ventricular mass index and end diastolic diameter and decreased LVEF. It is essential for the prognosis of heart failure. Recently, Kempf et al. (2007b) examined 455 CHF patients (average age, 64 years) and found that the GDF-15 level increased significantly and was positively correlated with CHF severity. In addition to NYHA function class, LVEF, and NT-proBNP level, GDF-15 provides prognostic information for CHF patients. GDF-15 can be used as an independent biomarker in CHF patients. Lok et al. (2012) reported that circulating GDF-15 was associated with myocardial fibrosis in patients with non-ischemic dilated cardiomyopathy, which decreased rapidly after left ventricular assist device support. Wollert and Kempf (2012) found that circulating GDF-15 rapidly decreases. They proposed that GDF-15 may be used to provide specific insight into peripheral disease pathways activated in patients with heart failure.

We found that the plasma GDF-15 level was positively related to NT-proBNP level and negatively related to LVEF. These results suggest that CHF patients have significantly increased GDF-15 levels compared to normal subjects, indicating that GDF-15 can be used as a biomarker for myocardial injury. We also found that the GDF-15 level in the subgroups of CHF patients, which were divided based on NYHA function class, was significantly higher than that in the control group. This confirmed the value of GDF-15 for CHF diagnosis. Furthermore, we found that the GDF-15 level in the CAD group was significantly higher than that in the control group. Combined with the area under the curve in the ROC curve, we suggest that GDF-15 may be used as a more efficient biomarker than NT-proBNP.

There were some limitations to this study, such as the small patient sample size. In addition, selection bias may have occurred because of the number of patients.

Our results suggest that GDF-15 level in CAD patients was significantly higher than that in the control group. GDF-15 increased more dramatically in CAD patients with CHF and was positively related to the NT-proBNP level. Because all patients were of Han nationality, our study provides novel insight into GDF-15. Our data indicate that GDF-15 may be a suitable biomarker for cardiovascular diseases. Further studies are necessary to confirm our results.

\section{REFERENCES}

Bootcov MR, Bauskin AR, Valenzuela SM, Moore AG, et al. (1997). MIC-1, a novel macrophage inhibitory cytokine, is a divergent member of the TGF-beta superfamily. Proc. Natl. Acad. Sci. U. S. A. 94: 11514-11519. 
Cheitlin MD, Armstrong WF, Aurigemma GP, Beller GA, et al. (2003). ACC/AHA/ASE 2003 guideline update for the clinical application of echocardiography - summary article: a report of the American College of Cardiology/ American Heart Association Task Force on Practice Guidelines (ACC/AHA/ASE Committee to Update the 1997 Guidelines for the Clinical Application of Echocardiography). J. Am. Coll. Cardiol. 42: 954-970.

de Boer RA, Lok DJ, Jaarsma T, van der Meer P, et al. (2011). Predictive value of plasma galectin-3 levels in heart failure with reduced and preserved ejection fraction. Ann. Med. 43: 60-68.

Goldsmith SR and Gheorghiade M (2005). Vasopressin antagonism in heart failure. J. Am. Coll. Cardiol. 46: 1785-1791.

Hunt SA (2005). ACC/AHA 2005 guideline update for the diagnosis and management of chronic heart failure in the adult: a report of the American College of Cardiology/American Heart Association Task Force on Practice Guidelines (Writing Committee to Update the 2001 Guidelines for the Evaluation and Management of Heart Failure). J. Am. Coll. Cardiol. 46: e1-e82.

Iqbal N, Alim KS, Aramin H, Iqbal F, et al. (2013). Novel biomarkers for heart failure. Expert Rev. Cardiovasc. Ther. 11: 1155-1169.

Kempf T and Wollert KC (2009). Growth differentiation factor-15: a new biomarker in cardiovascular disease. Herz 34: 594-599.

Kempf T, Eden M, Strelau J, Naguib M, et al. (2006). The transforming growth factor-beta superfamily member growthdifferentiation factor-15 protects the heart from ischemia/reperfusion injury. Circ. Res.98: 351-360.

Kempf T, Horn-Wichmann R, Brabant G, Peter T, et al. (2007a). Circulating concentrations of growth-differentiation factor 15 in apparently healthy elderly individuals and patients with chronic heart failure as assessed by a new immunoradiometric sandwich assay. Clin. Chem. 53: 284-291.

Kempf T, von Haehling S, Peter T, Allhoff T, et al. (2007b). Prognostic utility of growth differentiation factor-15 in patients with chronic heart failure. J. Am. Clin. Cardiol. 50: 1054-1060.

Kempf T, Sinning JM, Quint A, Bickel C, et al. (2009). Growth-differentiation factor-15 for risk stratification in patients with stable and unstable coronary heart disease: results from the AtheroGene study. Circ. Cardiovasc. Genet. 2: 286-292.

Kempf T, Zarbock A, Widera C, Butz S, et al. (2011). GDF-15 is an inhibitor of leukocyte integrin activation required for survival after myocardial infarction in mice. Nat. Med. 17: 581-588.

Khan SQ, Ng K, Dhillon O, Kelly D, et al. (2009). Growth differentiation factor-15 as a prognostic marker in patients with acute myocardial infarction. Eur. Heart J. 30: 1057-1065.

Lok DJ, Van Der Meer P, de la Porte PW, Lipsic E, et al. (2010). Prognostic value of galectin-3, a novel marker of fibrosis, in patients with chronic heart failure: data from the DEAL-HF study. Clin. Res. Cardiol.99: 323-328.

Lok DJ, Klip IT, Lok SI, Bruggink-André de la Porte PW, et al. (2013). Incremental prognostic power of novel biomarkers (growth-differentiation factor-15, high-sensitivity C-reactive protein, galectin-3, and high-sensitivity troponin-T) in patients with advanced chronic heart failure. Am. J. Cardiol. 112: 831-837.

Lok SI, Winkens B, Goldschmeding R, van Geffen AJ, et al. (2012). Circulating growth differentiation factor-15 correlates with myocardial fibrosis in patients with non-ischaemic dilated cardiomyopathy and decreases rapidly after left ventricular assist device support. Eur. J. Heart Fail. 14: 1249-1256.

Meeran K, O’Shea D, Upton PD, Small CJ, et al. (1997). Circulating adrenomedullin does not regulate systemic blood pressure but increases plasma prolactin after intravenous infusion in humans: a pharmacokinetic study. J. Clin. Endocrinol. Metab. 82: 95-100.

Meier-Ewert HK, Ridker PM, Rifai N, Regan MM, et al. (2004). Effect of sleep loss on C-reactive protein, an inflammatory marker of cardiovascular risk. J. Am. Coll. Cardiol. 43: 678-683.

Morgenthaler NG, Struck J, Alonso C and Bergmann A (2006). Assay for the measurement of copeptin, a stable peptide derived from the precursor of vasopressin. Clin. Chem. 52: 112-119.

Sanada S, Hakuno D, Higgins LJ, Schreiter ER, et al. (2007). IL-33 and ST2 comprise a critical biomechanically induced and cardioprotective signaling system. J. Clin. Invest. 117: 1538-1549.

Shah RV, Chen-Tournoux AA, Picard MH, van Kimmenade RR, et al. (2010). Galectin-3, cardiac structure and function, and long-term mortality in patients with acutely decompensated heart failure. Eur. J. Heart Fail. 12: 826-832.

Szinnai G, Morgenthaler NG, Berneis K, Struck J, et al. (2007). Changes in plasma copeptin, the c-terminal portion of arginine vasopressin during water deprivation and excess in healthy subjects. J. Clin. Endocrinol. Metab. 92: 3973-3978.

Tan M, Wang Y, Guan K and Sun Y (2000). PTGF-beta, a type beta transforming growth factor (TGF-beta) superfamily member, is a p53 target gene that inhibits tumor cell growth via TGF-beta signaling pathway. Proc. Natl. Acad. Sci. USA 97: 109-114.

Tsutamoto T, Hisanaga T, Wada A, Maeda K, et al. (1998). Interleukin-6 spillover in the peripheral circulation increases with the severity of heart failure, and the high plasma level of interleukin-6 is an important prognostic predictor in patients with congestive heart failure. J. Am. Coll. Cardio. 31: 391-398.

van Kimmenade RR, Januzzi JL Jr, Ellinor PT, Sharma UC, et al. (2006). Utility of amino-terminal pro-brain natriuretic 
peptide, galectin-3, and apelin for the evaluation of patients with acute heart failure. J. Am. Clin. Cardiol. 48: 1217-1224. von Haehling S, Filippatos GS, Papassotiriou J, Cicoira M, et al. (2010). Mid-regional pro-adrenomedullin as a novel predictor of mortality in patients with chronic heart failure. Eur. J. Heart Fail. 12: 484-491.

Wollert KC and Kempf T (2012). GDF-15 in heart failure: providing insight into end-organ dysfunction and its recovery? Eur. J. Heart Fail. 14: 1191-1193.

Wollert KC, Kempf T, Lagerqvist B, Lindahl B, et al. (2007a). Growth differentiation factor 15 for risk stratification and selection of an invasive treatment strategy in non ST-elevation acute coronary syndrome. Circulation 116: 15401548.

Wollert KC, Kempf T, Peter T, Olofsson S, et al. (2007b). Prognostic value of growth-differentiation factor-15 in patients with non-ST-elevation acute coronary syndrome. Circulation 115: 962-971.

Xu J, Kimball TR, Lorenz JN, Brown DA, et al. (2006). GDF15/MIC-1 functions as a protective and antihypertrophic factor released from the myocardium in association with SMAD protein activation. Circ. Res.98: 342-350. 